\title{
Subdiffusion in the Anderson model on the random regular graph
}

\author{
Giuseppe De Tomasi, ${ }^{1,2, *}$ Soumya Bera $\odot,{ }^{3}$ Antonello Scardicchio,,${ }^{4,5}$ and Ivan M. Khaymovich $\oplus^{6}$ \\ ${ }^{1}$ Department of Physics, T42, Technische Universität München, James-Franck-Straße 1, D-85748 Garching, Germany \\ ${ }^{2}$ T.C.M. Group, Cavendish Laboratory, JJ Thomson Avenue, Cambridge CB3 OHE, United Kingdom \\ ${ }^{3}$ Department of Physics, Indian Institute of Technology Bombay, Mumbai 400076, India \\ ${ }^{4}$ Abdus Salam International Center for Theoretical Physics, Strada Costiera 11, 34151 Trieste, Italy \\ ${ }^{5}$ INFN, Sezione di Trieste, Via Valerio 2, 34126 Trieste, Italy \\ ${ }^{6}$ Max-Planck-Institut für Physik komplexer Systeme, Nöthnitzer Straße 38, 01187 Dresden, Germany
}

(Received 11 September 2019; revised manuscript received 20 December 2019; accepted 10 January 2020; published 3 March 2020)

\begin{abstract}
We study the finite-time dynamics of an initially localized wave packet in the Anderson model on the random regular graph (RRG) and show the presence of a subdiffusion phase coexisting both with ergodic and putative nonergodic phases. The full probability distribution $\Pi(x, t)$ of a particle to be at some distance $x$ from the initial state at time $t$ is shown to spread subdiffusively over a range of disorder strengths. The comparison of this result with the dynamics of the Anderson model on $\mathbb{Z}^{d}$ lattices, $d>2$, which is subdiffusive only at the critical point implies that the limit $d \rightarrow \infty$ is highly singular in terms of the dynamics. A detailed analysis of the propagation of $\Pi(x, t)$ in space-time $(x, t)$ domain identifies four different regimes determined by the position of a wave front $X_{\text {front }}(t)$, which moves subdiffusively to the most distant sites $X_{\text {front }}(t) \sim t^{\beta}$ with an exponent $\beta<1$. Importantly, the Anderson model on the RRG can be considered as proxy of the many-body localization transition (MBL) on the Fock space of a generic interacting system. In the final discussion, we outline possible implications of our findings for MBL.
\end{abstract}

\section{DOI: 10.1103/PhysRevB.101.100201}

Introduction. The common belief that generic, isolated quantum systems thermalize as a result of their own dynamics has been challenged by a recent line of works showing that strong enough disorder can prevent them reaching thermal equilibrium [1,2]. This phenomenon, referred to as manybody localization (MBL) [1-7], generalizes the concept of Anderson localization [8] to the case of interacting particles, and has an important bearing on our understanding of quantum statistical mechanics.

Although MBL has been extensively studied [3,6,7], many of its aspects are still under intense debate. For example, only little is known on the nature of the MBL transition [9-13]. Recent numerical results show that the critical point of the transition may have been previously underestimated $[14,15]$ and critical exponents extracted with exact numerics seems to violate general constraints (i.e., so-called Harris bounds) $[3,16]$. Even the nature of the ergodic phase is not completely settled. For instance, subdiffusive dynamics has been observed on finite time scales and system sizes [17-24], but its mechanism and asymptotic limit are far from being clear [14,25-28].

Numerically these difficulties originate from the exponentially increasing complexity of the problem with system sizes,

\footnotetext{
*gd453@cam.ac.uk
}

Published by the American Physical Society under the terms of the Creative Commons Attribution 4.0 International license. Further distribution of this work must maintain attribution to the author(s) and the published article's title, journal citation, and DOI. Open access publication funded by the Max Planck Society. which makes the resolution of these open issues an extremely hard task. One way to overcome this problem is to consider approximate calculation methods like matrix product states [14,29-32] in order to increase significantly system sizes. Another way is to find proxies of interesting observables in more tractable models, which can reproduce the salient intrinsic features of MBL systems [33-38]. In this work we take the latter route considering an Anderson model on a hierarchical

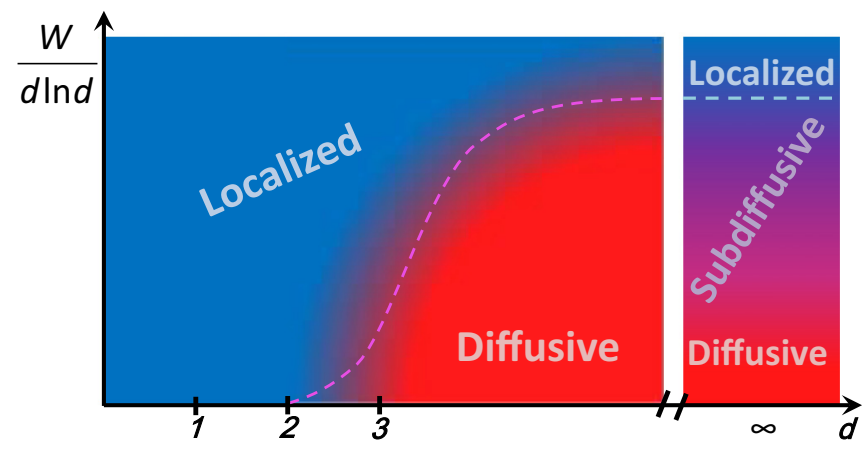

FIG. 1. Schematic phase diagram of the anomalous transport of the Anderson model in $d$ dimension. In $d=1,2$ the system is fully localized at any finite disorder. For $d>2$ the system has an Anderson transition at disorder strength $W=W_{\mathrm{AT}}$; for $W<W_{\mathrm{AT}}$ the transport is diffusive and subdiffusive only at the critical point. At small $d-2 \ll 1$ the critical disorder $W_{\mathrm{AT}} \sim(d-2)$ (linear behavior of dashed line), while at large $d$ it is given by $W_{\mathrm{AT}} \sim d \ln d$ (dashed line saturation). The limit $d \rightarrow \infty$ is given by the Anderson model on the RRG with the branching number $K$. The latter limit is characterized by three distinct phases: a diffusive, subdiffusive, and a localized one. 
treelike structure as a proxy for more realistic many-body systems. Indeed, in [1,39], the idea of mapping interacting disordered electrons to an effective Anderson model on a section of Fock space was used to give evidence of the stability of the MBL phase. Recently, this paradigm and the hierarchical structure of the Fock space of generic many-body systems have revived interest in the Anderson problem on locally treelike structures, such as the random regular graph (RRG).

Combining the hierarchical structure of the Fock space with the simplicity of regular graphs, i.e., the fixed branching number, RRG can be considered as a natural choice to approximate MBL systems [33-35] and hope to overcome some of the numerical difficulties that have been mentioned earlier. Apart from the fact that RRG gives a new emphasis on the field of Anderson localization, independently also its own physics is extremely rich [40-49]. For instance, it has been shown that there is a possibility of a nonergodic extended (NEE) phase composed of critical states and placed between the ergodic and the localized phase [33,41-44]. Nevertheless, it has been argued that this NEE phase might merely be a finite-size effect and would disappear in the thermodynamic limit [34,50-61]. However, this intricate question is far from being resolved.

Following the mapping of RRG to the Fock space of many-body systems, one expects the ergodic phase of wave functions on RRG to be qualitatively mapped to the validity range of the eigenstate thermalization hypothesis (ETH) [62-64] for many-body eigenstates. Furthermore, it has been recently suggested analytically and numerically confirmed that subleading corrections of ETH assumptions may lead to slow dynamics of local observables after quench instead of a diffusive one [17].

Motivated by the above-mentioned mapping, we study the spreading of an initially localized wave packet in the Anderson model on the RRG as a probe of different dynamical phases. In many-body systems, this can be considered as a proxy for the nonequilibrium dynamics of local operators after quench $[33,65,66]$ and also as a direct measure for entanglement propagation $[65,66]$. We give evidence of existence of subdiffusive dynamical phase over an entire range of parameters both in a part of the phase diagram where most of the works [34,42-47,50-61] agree on ergodic nature of eigenstates according to standard wave-function analysis and in a putative nonergodic phase [33,41-44]. Moreover, it is important to point out that the dynamics of the Anderson model on $\mathbb{Z}^{d}, d>2$, is believed to be diffusive within its ergodic phase and subdiffusive only at the critical point $[67,68]$. Thus the found subdiffusive phase in the limiting dimension $d \rightarrow$ $\infty$ of the RRG provides a further example of the importance of dimensionality in the physics of localization, beside the well known example of fully localized systems in $d=1,2$; see Fig. 1.

Model and methods. The Anderson model on the RRG is defined as

$$
\hat{H}=-\sum_{\substack{x, y \\ x \sim y}}^{L}|x\rangle\left\langle y\left|+\sum_{x}^{L} h_{x}\right| x\right\rangle\langle x|,
$$

where $x$ counts $L$ site states $|x\rangle$ on the RRG. The first sum in $\hat{H}$ runs over sites $(x, y)$ that are connected $(x \sim y)$ on the RRG with fixed branching number (the number of neighbors of each site is fixed to $K+1=3$ ). $\left\{h_{x}\right\}$ independent random variables distributed uniformly between $[-W / 2, W / 2]$. This model is known to have an Anderson localization transition at $W_{\mathrm{AT}} \approx 18.1 \pm 0.1[34,44,48]$.

We are interested in studying the full propagation of a wave function initially localized in a neighborhood of a site state $\left|x_{0}\right\rangle$, and having energy concentrated in a window of size $\delta E$ around the center of the band, $E=0$.

A standard description for the dynamics employs the distribution function $\Pi(x, t)[69]$ which determines the probability to find the particle at time $t$ in some state at distance $x$ from the initial one

$$
\Pi(x, t)=\frac{\overline{\sum_{y: d\left(y, x_{0}\right)=x}\left|\left\langle y\left|\hat{P}_{\Delta E} e^{-i \hat{H} t} \hat{P}_{\Delta E}\right| x_{0}\right\rangle\right|^{2}}}{\sum_{y}\left|\left\langle y\left|\hat{P}_{\Delta E}\right| x_{0}\right\rangle\right|^{2}} .
$$

The sum in Eq. (2) runs over all states $|y\rangle$ located at distance $d\left(y, x_{0}\right)=x$ from the initial state $\left|x_{0}\right\rangle$. The distance $d\left(y, x_{0}\right)$ is defined as the shortest path's length that connects two sites on the RRG. Importantly, in the many-body setting this distance is related to the Hamming metric of the Fock space $[39,65,66,70]$. The computation of the Hamming distance between two Fock states involves only the measure of local observables, and has been measured experimentally in the MBL context, specially using it as a witness for entanglement propagation [65].

The overline in Eq. (2) indicates the average over disorder, graph ensemble, and initial states $\left|x_{0}\right\rangle . \hat{P}_{\Delta E}=\sum_{E \in \Delta E}|E\rangle\langle E|$ is the projector onto eigenstates of $\hat{H}$ with energy $E$ from a small energy shell $E \in \Delta E=[-\delta E / 2, \delta E / 2]$ around the middle of the spectrum of $\hat{H}$. In particular, we consider $\delta E$ to be a small fraction $f(f=1 / 8)$ of the entire bandwidth $E_{B W}$ $\left(\delta E=f E_{B W}\right)$.

The usage of the projector is motivated by several reasons. First, $\hat{P}_{\Delta E}$ avoids the localized eigenstates at the edge of the spectrum [71]. Second, the initialization of the system in the microcanonical state with well-defined energy $E \in \Delta E$ in a small interval in the middle of the spectrum mimics ETH assumptions of many-body physics and under otherwise equal conditions prefers thermalization. Thus slow nondiffusive propagation of such projected wave packet should rule out the possibility of a fully ergodic phase (equivalent to random matrix theory [72]). Finally, the projector can be used as a dynamical indicator to distinguish a fully ergodic system from a nonergodic one $[73,74]$. In a fully ergodic phase, as a consequence of level repulsion, the return probability, $\Pi(0, t)$, takes a standard form [74] given by

$$
\frac{\Pi(0, t)}{\Pi(0,0)}=\left(\frac{\sin \delta E t}{\delta E t}\right)^{2} .
$$

The projector $\hat{P}_{\Delta E}$ slightly spreads the initial deltafunction-like state $\left|x_{0}\right\rangle$ to the wave packet $\hat{P}_{\Delta E}\left|x_{0}\right\rangle$ with a finite width. This initialization supports the semiclassical description of wave-packet propagation in the system. We ensure that our results do not change significantly with $\delta E$, provided it is not too big [75].

As a further measure of the spread of the wave packet, we study the first moment of $\Pi(x, t)$,

$$
X(t)=\sum_{x} x \Pi(x, t) .
$$




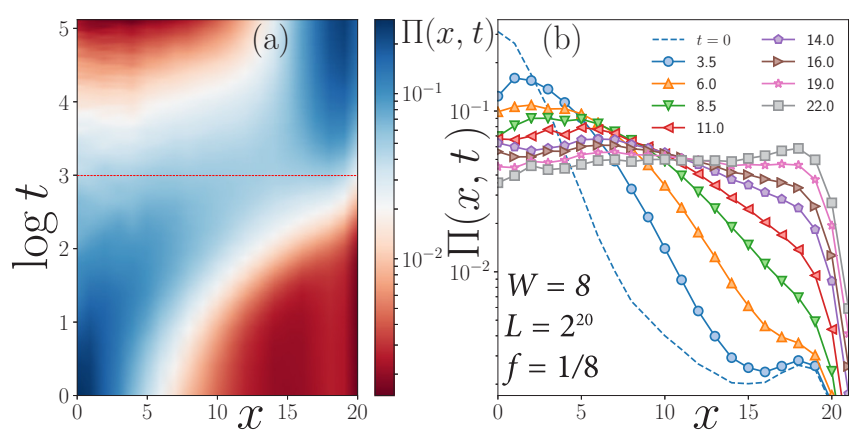

FIG. 2. Probability $\Pi(x, t)$ for the particle to be at distance $x$ from the initial state at time $t$. (a) $\Pi(x, t)$ versus distance $x$ and time $t$ in a color plot. Blue (red) color corresponds to high (low) values of $\Pi(x, t)$ and shows the propagation of the initially localized wave packet with the initial size $X(0) \simeq 2$ through the uniform distribution over distance $\Pi\left(x, t_{\mathrm{Th}}\right) \simeq$ const (see the dashed line $t_{\mathrm{Th}} \approx 22$ ) to the uniform distribution over sites $\Pi(x, \infty) \simeq \mathcal{N}(x) / L$. (b) Cross section of the color plot in panel (a) at several times below $t_{\mathrm{Th}}$, showing how the wave front propagates to the diameter of the graph. All plots are shown at the most representative disorder amplitude $W=8$ for fixed system size $L=2^{20}$.

The wave-packet width at time $t=0$ induced by $\hat{P}_{\Delta E}$ can be simply estimated by $X(0)=\sum_{x} x \Pi(x, 0)$.

In Ref. [73] we have shown that for small values of $W$ $\left(0<W<0.16 W_{\mathrm{AT}} \simeq 3\right)$ the return probability $\Pi(0, t)$ is consistent with the result of Eq. (3), confirming that the system is in a fully ergodic phase [76,77]. For larger disorder, $W \in$ $\left[0.4 W_{\mathrm{AT}}, 0.7 W_{\mathrm{AT}}\right] \simeq[8,13], \Pi(0, t)$ decays as a stretched exponential $\sim e^{-\Gamma t^{\beta(W)}}$, where the exponent is well approximated by

$$
\beta(W) \simeq 1-W / W_{\mathrm{AT}}, \quad 0.4 W_{\mathrm{AT}} \lesssim W \lesssim 0.7 W_{\mathrm{AT}}
$$

and goes to zero at the Anderson transition. As a consequence, the drastic change in the time evolution of $\Pi(0, t)$ gives evidence of the existence of two dynamically distinct phases.

As a side remark, before coming to the results, we stress the difference between the wave-packet propagation on hierarchical structures and $d$-dimensional lattices like $\mathbb{Z}^{d}$. It is well known that the return probability for a classical unbiased random walk on a Bethe lattice with branching number $K$ decays exponentially fast in time $\sim e^{-\Omega(K) t}$ [78] due to the exponential growth $K^{x}$ of the number of sites with the distance $x$ from an initial point $\left|x_{0}\right\rangle$. Instead, in $\mathbb{Z}^{d}$ lattices the typical behavior is diffusive $\sim t^{-d / 2}$ as the number of sites at distance $x$ grows algebraically $\mathcal{N}(x) \sim x^{d-1}$. Thus the diffusive propagation on hierarchical tree lattices is characterized by a linear growth of the width of the wave packet with time $X(t) \sim t$ [see Eq. (4)] unlike $X(t) \sim t^{1 / 2}$ in $d$-dimensional lattices. Noticing this difference, we call the propagation in RRG subdiffusive if $X(t) \sim t^{\beta}$ with $\beta<1$.

In this work, we show that, as time increases, $\Pi(x, t)$ relaxes forming a wave front $X_{\text {front }}(t)$ that moves subdiffusively to the most distant sites, as shown in Fig. 2. More specifically the propagation of $\Pi(x, t)$ can be divided into four regions in space-time domain $(x, t)$ depending on the position of the moving front $X_{\text {front }}(t)$.

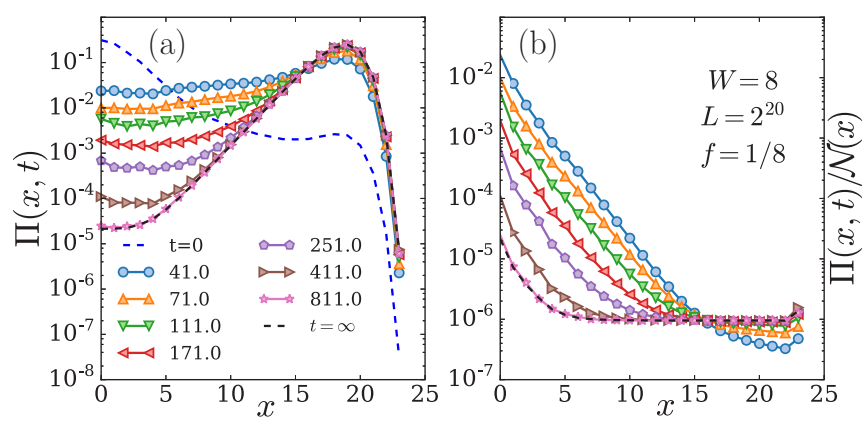

FIG. 3. Probability $\Pi(x, t)$ versus distance $x$ at large times $t>$ $t_{\mathrm{Th}}$. (a) The cross sections of Fig. 2(a) at several times above $t_{\mathrm{Th}}$, when the propagation front has already reached the diameter of the graph $X_{\text {front }}>D=\ln L / \ln K . \Pi(x, t)$ relaxes from the uniform distribution in the distance $\Pi\left(x, t_{\mathrm{Th}}\right) \simeq$ const to the uniform distribution over sites $\Pi(x, \infty) \simeq \mathcal{N}(x) / L$. Dashed line shows the initial distribution $\Pi(x, 0)$ as a guide for eyes. (b) The distribution from panel (a) renormalized by the mean number of sites $\mathcal{N}(x)$ at some distance $x$ from an initial site state $\left|x_{0}\right\rangle$. This figure gives evidence of the space-time factorization Eq. (8), once the front has already passed, $X_{\text {front }}(t)>D$. The parameters are the same as in Fig. 2.

(i) At large distances (small times), $x>X_{\text {front }}(t)$, the wave front has not yet reached $x$, and the distribution is nearly unperturbed

$$
\Pi(x, t) \approx \Pi(x, 0), \quad x>X_{\text {front }}(t)
$$

(see the red area at small times in Fig. 2 and the plateau at short times in the inset of Fig. 4).

(ii) At $x \simeq X_{\text {front }}(t)$ in proximity of the front propagation, $\Pi\left(x \lesssim X_{\text {front }}(t), t\right)$ renormalized by its maximal value $\Pi\left(X_{\text {front }}(t), t\right)$ collapses to a universal function

$$
\Pi(x, t)-\Pi(x, \infty)=\Pi\left(X_{\text {front }}(t), t\right) f\left(X_{\text {front }}(t)-x\right)
$$

with the semiclassical $(x, t)$ front propagation governed by the parameter $X_{\text {front }}(t)-x$, as shown in Fig. 4(b).

In particular, the front moves subdiffusively, $X_{\text {front }}(t) \sim$ $t^{\beta(W)}$, where $\beta(W)$ is given by Eq. (5) [79].

(iii) At larger times (smaller distances within the wave packet), $x<X_{\text {front }}(t), \Pi(x, t)$ shows space-time factorization

$$
\Pi(x, t)-\Pi(x, \infty)=g(x)[\Pi(0, t)-\Pi(0, \infty)],
$$

with respect to the return probability $\Pi(0, t)$ and a certain function $g(x)$, as shown in Figs. 3 and 4(a). Thus, in this regime, the relaxation is dictated by the return probability which is connected to the front of propagation by the following relation:

$$
\Pi(0, t) \sim \exp \left[-\lambda X_{\text {front }}(t)\right], \quad \lambda>0 .
$$

(iv) Eventually at very long times $\Pi(x, t)$ saturates at the uniform distribution over sites $\Pi(x, \infty)=\mathcal{N}(x) / L$, where $\mathcal{N}(x) \sim K^{x}$ is the mean number of sites at some distance $x$ from an initial site state $\left|x_{0}\right\rangle$ and $L$ is the number of sites; see Fig. 3.

Stages (i) and (ii) are presented only for times $t<t_{\mathrm{Th}}$ corresponding to front propagation inside the graph $X_{\text {front }}(t)<$ $D$, where $D \simeq \ln L / \ln K$ is the diameter of the graph. At larger times only relaxation with the return probability (iii) and saturation (iv) stages are relevant. 

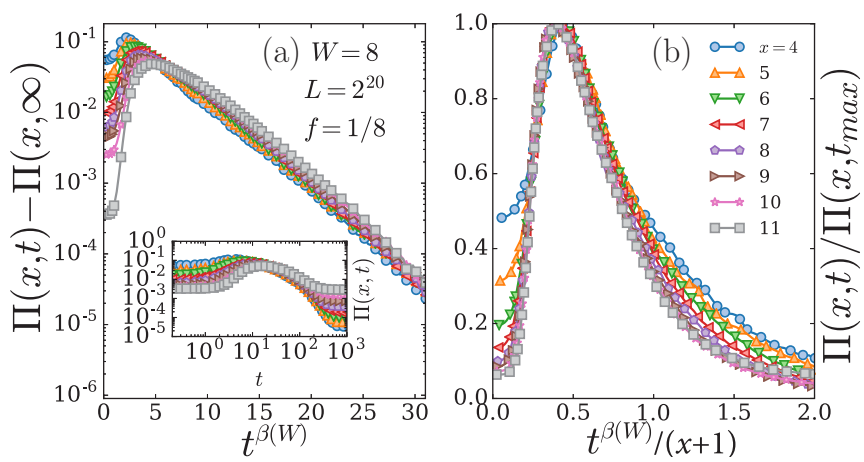

FIG. 4. Collapse of the probability $\Pi(x, t)$ versus $t$ at different distances $x$. (a) Space-time factorization of $\Pi(x, t)$ Eq. (8) at large times $t>t_{\mathrm{Th}}(x)$ corresponding to the relaxation inside the wave packet $x<X_{\text {front }}(t)$. This relaxation is proportional to the return probability $\Pi(0, t) \sim e^{-\Gamma t^{\beta(W)}}$. The time axis is properly rescaled with the power $\beta(W)=1-W / W_{\mathrm{AT}}$, Eq. (5), to emphasize stretched exponential decay of $\Pi(x, t)$. The inset shows $\Pi(x, t)$ on a log-log scale for all four stages of the evolution: initial distribution $\Pi(x, t) \approx$ $\Pi(x, 0)$ before wave front coming $x>X_{\text {front }}(t)$, the maximum, the common tail after front passing, and the eventual saturation. (b) Collapse of $\Pi(x, t)$ Eq. (7) around the wave front confirming subdiffusive propagation $X_{\text {front }}(t) \sim t^{\beta(W)}$. The parameters are the same as in Fig. 2.

Results. We focus our attention on intermediate disorder values $8 \leqslant W \leqslant 14$, for which the return probability shows slow dynamics $\Pi(0, t) \sim e^{-\Gamma t^{\beta(W)}}$, with $\beta(W)$ given by Eq. (5) [80]. The propagation of $\Pi(x, t)$ versus distance $x$ and time $t$ at fixed system size $L=2^{20} \approx 10^{6}$ is shown in Fig. 2(a). At small times, $\delta E t \sim O(1)$, the wave-packet width is small $X(0) \sim 2$, Eq. (4). As time evolves, the wave packet spreads in the form of wave-front $X_{\text {front }}(t)$ [81] which transfers most of its weight to the most distant sites $[X(\infty) \simeq D]$, as shown in Fig. 2.

Although the dynamics on the RRG is typically not isotropic, the time scale $t_{\mathrm{Th}}$ at which the wave front reaches the diameter could be seen as a natural choice for the Thouless time analogous to the time that a charge needs to propagate through a diffusive conductor [82]. The wave-front propagation at times $t<t_{\mathrm{Th}}, X_{\text {front }}(t)<D$, can be seen in Fig. 2(a) and is explicitly shown in Fig. 2 (b). Already for time $t_{\mathrm{Th}} \approx 22$, as emphasized in the color plot of Fig. 2(a) (red dashed line), the main core has lost most of its amplitude $\Pi\left(x \leqslant 5, t_{\mathrm{Th}}\right) / \Pi(x \leqslant$ $5,0) \sim 10^{-1}$ and $\Pi(x, t)$ becomes nearly uniform over the distance, $\Pi\left(x, t_{\mathrm{Th}}\right) \simeq$ const.

Figure 3(a) shows $\Pi(x, t)$ as a function of $x$ at large times, $t>t_{\mathrm{Th}}$, when the front has already reached the diameter of the graph. In this regime, $\Pi(x, t)$ relaxes uniformly in distance $x$ to the equiprobable configuration on the graph $\Pi(x, \infty)=\frac{\mathcal{N}(x)}{L}$ [dashed line in Fig. 3(a)]. Thus, at these times, $\Pi(x, t)-\Pi(x, \infty)$ is factorized in $(x, t)$ according to Eq. (8), with $g(0)=1$ due to the uniform relaxation seen as well for $x=0$.

Detailed analysis shows that the factorization works beyond the limit, $t>t_{\mathrm{Th}}$, provided the wave front crossed the observation point $x<X_{\text {front }}(t)$; see Fig. 4(a). Subtracting from $\Pi(x, t)$ its long time limit $\Pi(x, \infty)$ results in the collapse in


FIG. 5. Subdiffusive wave-packet spreading. (a) Wave-packet width $X(t)=\sum_{x} x \Pi(x, t)$ versus time $t$ on a log-log scale for different disorder strengths supplemented by a guide for eyes $\sim t^{\beta(W)}$ (dashed lines), with $\beta(W)=1-W / W_{\mathrm{AT}}$, Eq. (5). (b) Collapse of wave-packet width $\ln X(t) / \beta(W)$ from panel (a) showing the unit slope versus $t$ (dashed line) in increasing time interval with growing disorder amplitude $W$. The parameters are the same as in Fig. 2.

Eq. (8) of the curves for any $x<X_{\text {front }}(t)$. It is important to note that in Fig. 4 the time axis is rescaled as $t^{\beta(W)}$, with $\beta(W)$ given by Eq. (5) to emphasize the stretched-exponential time relaxation shown to be true for the return probability in Ref. [73], $\Pi(0, t)-\Pi(0, \infty) \simeq e^{-\Gamma t^{\beta(W)}}$. Moreover, raw $\Pi(x, t)$ is shown in the inset of Fig. 4(a) on a log-log scale to demonstrate nearly unperturbed short-time behavior of $\Pi(x, t)$ in Eq. (6).

In order to analyze the time dependence of the wave-front propagation $X_{\text {front }}(t)$ in Fig. 4(b) we collapse the curves dividing $\Pi(x, t)$ by its maximum, $\Pi\left(X_{\text {front }}(t), t\right)$, and rescale the time $t^{\beta(W)}$ in order to collapse the position of the maximum. This collapse allows us to extract the following subdiffusive wave-front evolution:

$$
X_{\text {front }}(t) \simeq \Gamma(W) t^{\beta(W)}, \quad \beta(W)<1
$$

Moreover, the collapse of the curves, Fig. 4(b), implies the simple exponential dependence, Eq. (9), of the return probability versus $X_{\text {front }}(t)$ with a certain decay rate $\lambda, f(z)=e^{-\lambda z}$, Eq. (7) [83]. The front-propagation collapse, Eq. (7), is shown to work also for different disorder strengths in the range of interest $8 \leqslant W \leqslant 13[80]$.

As a further consequence, the Thouless time, defined as the time when the wave front reaches the graph diameter, scales as $t_{\mathrm{Th}} \sim\left(\frac{\ln L}{K}\right)^{1 / \beta(W)}$. The similar scaling of the Thouless time calculated for MBL systems in the subdiffusive phase [84,85] supports the idea that wave-packet dynamics on RRG is a good proxy for MBL systems.

Finally, we analyze the first moment $X(t)$, Eq. (4), of the radial probability distribution $\Pi(x, t)$. Figure 5 (a) shows the algebraic growth of $X(t)$ in time for several $W$

$$
X(t) \sim t^{\beta(W)},
$$

with the same subdiffusive exponent $\beta(W)$, Eq. (5), as in the wave-front propagation $X_{\text {front }}(t)$. Furthermore, the curves $X(t)$ can be reasonably well collapsed for the range of disorder 
strengths by considering the rescaled function $\frac{\ln X(t)}{\beta(W)}$ versus $\ln t$. This result is checked to be robust with respect to the finite-size effects and to the variation of the fraction $f$ [80].

Conclusion and discussions. In this work, we provide evidence of the existence of a subdiffusive phase for a finite range of parameters by probing the dynamics of an initially localized particle on the RRG via the probability distribution $\Pi(x, t)$ to detect it at distance $x$ at time $t$.

The relaxation of $\Pi(x, t)$ is characterized by the formation of a semiclassical wave front $X_{\text {front }}(t)$, moving subdiffusively to the most distant sites. Remarkably, as soon as the wave front passed the observation point $x$, the space-time factorization, $\Pi(x, t)=g(x) h(t)$, is found.

The Anderson model on RRG gives the first example of an entire subdiffusive phase as the systems on $\mathbb{Z}^{d}$ lattices are either localized for any finite disorder in $d=1,2$ or show subdiffusion only at the critical point, $d>2[67,68]$.

It is important to note that the existence of a subdiffusive phase is not in contrast with the possibility that the eigenfunctions are ergodic in terms of the inverse participation ratio (IPR) scaling as the inverse of the volume. To emphasize further, the IPR scaling is a statement about the nature of the fluctuations of the eigenfunctions equivalent to the long time limit $(t \rightarrow \infty)$ of certain dynamical observables. On the other hand, in our study we probe the time evolution of a wave packet and far away from the aforementioned limit. Thus our study excludes the scenario that the system is fully ergodic at $W \geqslant 8$, which is a stronger requirement than just IPR ergodicity discussed above [86].

We have to mention that some works $[61,87]$ claim only diffusive propagation $[\beta(W)=1]$ for all $W<W_{\mathrm{AT}}$; however, in the mathematically rigorous work [87], an absolutely continuous spectrum is assumed, which may not be so. We do not report any crossover to diffusivity for our available system sizes and time scales. Although we cannot completely rule out this possibility in the thermodynamic limits $L \rightarrow \infty$ and $t \rightarrow \infty$, the above finite-time subdiffusive dynamics is highly relevant for corresponding experiments in many-body systems.

In addition, the Anderson model on the RRG can be considered as a proxy for the dynamics of more realistic MBL systems. Our finding thus opens the possibility to have a subdiffusive dynamical phase in Fock space, that might imply slow relaxation of local observables. This possible implication of subdiffusive spatial dynamics in MBL systems [17-22,25] from slow Fock space dynamics may give rise to a different mechanism which does not invoke the existence of Griffiths effects [11,12,22]. Recent works (see, e.g., Ref. [88]) show that in MBL systems the subdiffusive phase is also consistent with a weakly ergodic phase confirming RRG as the commonly believed proxy.

We thank V. E. Kravtsov, F. Pollmann, and S. Warzel for helpful and stimulating discussions and M. Haque and P. A. McClarty for careful reading of the manuscript. S.B. acknowledges support from DST, India, through Ramanujan Fellowship Grant No. SB/S2/RJN-128/2016 and Early Career Research Award No. ECR/2018/000876; S.B. and G.D.T. also thank the visitor program of the Max Planck Institute for the Physics of Complex Systems, Dresden for hospitality during the final stage of the work. I.M.K. acknowledges the support of German Research Foundation (DFG) Grant No. KH 425/11 and the Russian Foundation for Basic Research Grant No. 17-52-12044.
[1] D. M. Basko, I. L. Aleiner, and B. L. Altshuler, Metal-insulator transition in a weakly interacting many-electron system with localized single-particle states, Ann. Phys. (NY) 321, 1126 (2006).

[2] I. V. Gornyi, A. D. Mirlin, and D. G. Polyakov, Interacting Electrons in Disordered Wires: Anderson Localization and Low- $t$ Transport, Phys. Rev. Lett. 95, 206603 (2005).

[3] R. Nandkishore and D. A. Huse, Many-body localization and thermalization in quantum statistical mechanics, Annu. Rev. Condens. Matter Phys. 6, 15 (2015).

[4] J. Z Imbrie, V. Ros, and A. Scardicchio, Local integrals of motion in many-body localized systems, Ann. Phys. (NY) 529, 1600278 (2017).

[5] D. A Abanin and Z. Papić, Recent progress in many-body localization, Ann. Phys. (NY) 529, 1700169 (2017).

[6] F. Alet and N. Laflorencie, Many-body localization: An introduction and selected topics, C. R. Phys. 19, 498 (2018), Quantum simulation / Simulation quantique.

[7] D. A. Abanin, E. Altman, I. Bloch, and M. Serbyn, Colloquium: Many-body localization, thermalization, and entanglement, Rev. Mod. Phys. 91, 021001 (2019).

[8] P. Anderson, Absence of diffusion in certain random lattices, Phys. Rev. 109, 1492 (1958).
[9] R. Vosk, D. A. Huse, and E. Altman, Theory of the Many-body Localization Transition in One-dimensional Systems, Phys. Rev. X 5, 031032 (2015).

[10] E. Altman and R. Vosk, Universal dynamics and renormalization in many-body-localized systems, Annu. Rev. Condens. Matter Phys. 6, 383 (2015).

[11] K. Agarwal, S. Gopalakrishnan, M. Knap, M. Müller, and E. Demler, Anomalous Diffusion and Griffiths Effects Near the Many-body Localization Transition, Phys. Rev. Lett. 114, 160401 (2015).

[12] V. Khemani, S. P. Lim, D. N. Sheng, and D. A. Huse, Critical Properties of the Many-body Localization Transition, Phys. Rev. X 7, 021013 (2017).

[13] F. Pietracaprina, G. Parisi, A. Mariano, S. Pascazio, and A. Scardicchio, Entanglement critical length at the many-body localization transition, J. Stat. Mech.: Theory Exp. (2017) 113102.

[14] E. V. H. Doggen, F. Schindler, K. S. Tikhonov, A. D. Mirlin, T. Neupert, D. G. Polyakov, and I. V. Gornyi, Many-body localization and delocalization in large quantum chains, Phys. Rev. B 98, 174202 (2018).

[15] J. Šuntajs, J. Bonča, T. Prosen, and L. Vidmar, Quantum chaos challenges many-body localization, arXiv:1905.06345. 
[16] A. Chandran, C. R. Laumann, and V. Oganesyan, Finite size scaling bounds on many-body localized phase transitions, arXiv: 1509.04285 .

[17] D. J. Luitz and Y. Bar Lev, Anomalous Thermalization in Ergodic Systems, Phys. Rev. Lett. 117, 170404 (2016).

[18] Y. Bar Lev, G. Cohen, and D. R. Reichman, Absence of Diffusion in an Interacting System of Spinless Fermions on a One-dimensional Disordered Lattice, Phys. Rev. Lett. 114, 100601 (2015).

[19] P. Prelovšek and J. Herbrych, Self-consistent approach to manybody localization and subdiffusion, Phys. Rev. B 96, 035130 (2017).

[20] V. K. Varma, A. Lerose, F. Pietracaprina, J. Goold, and A. Scardicchio, Energy diffusion in the ergodic phase of a many body localizable spin chain, J. Stat. Mech. (2017) 053101.

[21] M. Žnidarič, A. Scardicchio, and V. K. Varma, Diffusive and Subdiffusive Spin Transport in the Ergodic Phase of a Many-body Localizable System, Phys. Rev. Lett. 117, 040601 (2016).

[22] S. Gopalakrishnan, K. Agarwal, E. A. Demler, D. A. Huse, and M. Knap, Griffiths effects and slow dynamics in nearly manybody localized systems, Phys. Rev. B 93, 134206 (2016).

[23] I. Khait, S. Gazit, N. Y. Yao, and A. Auerbach, Spin transport of weakly disordered Heisenberg chain at infinite temperature, Phys. Rev. B 93, 224205 (2016).

[24] M. Schulz, S. Taylor, A. Scardicchio, and M. Znidaric, Phenomenology of anomalous transport in disordered onedimensional systems, arXiv:1909.09507.

[25] S. Bera, G. De Tomasi, F. Weiner, and F. Evers, Density Propagator for Many-body Localization: Finite-size Effects, Transient Subdiffusion, and Exponential Decay, Phys. Rev. Lett. 118, 196801 (2017).

[26] S. Gopalakrishnan, M. Müller, V. Khemani, M. Knap, E. Demler, and D. A. Huse, Low-frequency conductivity in manybody localized systems, Phys. Rev. B 92, 104202 (2015).

[27] F. Weiner, F. Evers, and S. Bera, Slow dynamics and strong finite-size effects in many-body localization with random and quasiperiodic potentials, Phys. Rev. B 100, 104204 (2019).

[28] G. De Tomasi, D. Hetterich, P. Sala, and F. Pollmann, Dynamics of strongly interacting systems: From Fock-space fragmentation to many-body localization, Phys. Rev. B 100, 214313 (2019).

[29] V. Khemani, F. Pollmann, and S. L. Sondhi, Obtaining Highly Excited Eigenstates of Many-body Localized Hamiltonians by the Density Matrix Renormalization Group Approach, Phys. Rev. Lett. 116, 247204 (2016).

[30] A. Chandran, J. Carrasquilla, I. H. Kim, D. A. Abanin, and G. Vidal, Spectral tensor networks for many-body localization, Phys. Rev. B 92, 024201 (2015).

[31] X. Yu, D. Pekker, and B. K. Clark, Finding Matrix Product State Representations of Highly Excited Eigenstates of Many-body Localized Hamiltonians, Phys. Rev. Lett. 118, 017201 (2017).

[32] S. P. Lim and D. N. Sheng, Many-body localization and transition by density matrix renormalization group and exact diagonalization studies, Phys. Rev. B 94, 045111 (2016).

[33] G. Biroli and M. Tarzia, Delocalized glassy dynamics and many-body localization, Phys. Rev. B 96, 201114(R) (2017).

[34] K. S. Tikhonov and A. D. Mirlin, Critical behavior at the localization transition on random regular graphs, Phys. Rev. B 99, 214202 (2019).
[35] V. Avetisov, A. Gorsky, S. Nechaev, and O. Valba, Localization and non-ergodicity in clustered random networks, J. Comp. Netw., cnz026 (2019).

[36] D. E. Logan and S. Welsh, Many-body localization in Fock space: A local perspective, Phys. Rev. B 99, 045131 (2019).

[37] S. Roy, D. E. Logan, and J. T. Chalker, Exact solution of a percolation analog for the many-body localization transition, Phys. Rev. B 99, 220201(R) (2019).

[38] S. Roy, J. T. Chalker, and D. E. Logan, Percolation in Fock space as a proxy for many-body localization, Phys. Rev. B 99, 104206 (2019).

[39] B. L. Altshuler, Y. Gefen, A. Kamenev, and L. S. Levitov, Quasiparticle Lifetime in a Finite System: A Nonperturbative Approach, Phys. Rev. Lett. 78, 2803 (1997).

[40] R. Abou-Chacra, D. J. Thouless, and P. W. Anderson, A selfconsistent theory of localization, J. Phys. C: Solid State Phys. 6, 1734 (1973).

[41] A. De Luca, B. L. Altshuler, V. E. Kravtsov, and A. Scardicchio, Anderson Localization on the Bethe Lattice: Nonergodicity of Extended States, Phys. Rev. Lett. 113, 046806 (2014).

[42] B. L. Altshuler, E. Cuevas, L. B. Ioffe, and V. E. Kravtsov, Nonergodic Phases in Strongly Disordered Random Regular Graphs, Phys. Rev. Lett. 117, 156601 (2016).

[43] B. L. Altshuler, L. B. Ioffe, and V. E. Kravtsov, Multifractal states in self-consistent theory of localization: Analytical solution, arXiv:1610.00758.

[44] V. E. Kravtsov, B. L. Altshuler, and L. B. Ioffe, Non-ergodic delocalized phase in Anderson model on Bethe lattice and regular graph, Ann. Phys. (NY) 389, 148 (2018).

[45] G. Biroli, G. Semerjian, and M. Tarzia, Anderson model on Bethe lattices: density of states, localization properties and isolated eigenvalue, Prog. Theor. Phys. Suppl. 184, 187 (2010).

[46] I. García-Mata, O. Giraud, B. Georgeot, J. Martin, R. Dubertrand, and G. Lemarié, Scaling Theory of the Anderson Transition in Random Graphs: Ergodicity and Universality, Phys. Rev. Lett. 118, 166801 (2017).

[47] I. García-Mata, J. Martin, R. Dubertrand, O. Giraud, B Georgeot, and G. Lemarié, Two critical localization lengths in the Anderson transition on random graphs, Phys. Rev. Research 2 012020(R) (2020).

[48] G. Parisi, S. Pascazio, F. Pietracaprina, V. Ros, and A. Scardicchio, Anderson transition on the Bethe lattice: An approach with real energies, J. Phys. A: Math. Theor. 53, 014003 (2020).

[49] S. Savitz, C. Peng, and G. Refael, Anderson localization on the Bethe lattice using cages and the Wegner flow, Phys. Rev. B 100, 094201 (2019).

[50] G. Biroli, A. C. Ribeiro-Teixeira, and M. Tarzia, Difference between level statistics, ergodicity and localization transitions on the Bethe lattice, arXiv:1211.7334.

[51] G. Biroli and M. Tarzia, Delocalization and ergodicity of the Anderson model on Bethe lattices, arXiv:1810.07545.

[52] F. L. Metz and I. Perez Castillo, Large Deviation Function for the Number of Eigenvalues of Sparse Random Graphs Inside an Interval, Phys. Rev. Lett. 117, 104101 (2016).

[53] F. L. Metz and I. P. Castillo, Level compressibility for the Anderson model on regular random graphs and the eigenvalue statistics in the extended phase, Phys. Rev. B 96, 064202 (2017) 
[54] M. Aizenman and S. Warzel, Extended States in a Lifshitz Tail Regime for Random Schrödinger Operators on Trees, Phys. Rev. Lett. 106, 136804 (2011).

[55] M. W. S. Aizenman, Resonant delocalization for random Schrödinger operators on tree graphs, J. Eur. Math. Soc. 15, 1167 (2013).

[56] V. Bapst, The large connectivity limit of the Anderson model on tree graphs, J. Math. Phys. 55, 092101 (2014).

[57] A. D. Mirlin and Y. V. Fyodorov, Localization transition in the Anderson model on the Bethe lattice: Spontaneous symmetry breaking and correlation functions, Nucl. Phys. B 366, 507 (1991).

[58] K. S. Tikhonov, A. D. Mirlin, and M. A. Skvortsov, Anderson localization and ergodicity on random regular graphs, Phys. Rev. B 94, 220203(R) (2016).

[59] K. S. Tikhonov and A. D. Mirlin, Fractality of wave functions on a Cayley tree: Difference between tree and locally treelike graph without boundary, Phys. Rev. B 94, 184203 (2016).

[60] M. Sonner, K. S. Tikhonov, and A. D. Mirlin, Multifractality of wave functions on a Cayley tree: From root to leaves, Phys. Rev. B 96, 214204 (2017).

[61] K. S. Tikhonov and A. D. Mirlin, Statistics of eigenstates near the localization transition on random regular graphs, Phys. Rev. B 99, 024202 (2019).

[62] M. Srednicki, Chaos and quantum thermalization, Phys. Rev. E 50, 888 (1994).

[63] J. M. Deutsch, Quantum statistical mechanics in a closed system, Phys. Rev. A 43, 2046 (1991).

[64] M. Rigol and M. Srednicki, Alternatives to Eigenstate Thermalization, Phys. Rev. Lett. 108, 110601 (2012).

[65] J. Smith, A. Lee, P. Richerme, B. Neyenhuis, P. W. Hess, P. Hauke, M. Heyl, D. A. Huse, and C. Monroe, Many-body localization in a quantum simulator with programmable random disorder, Nat. Phys. 12, 907 (2016).

[66] P. Hauke and M. Heyl, Many-body localization and quantum ergodicity in disordered long-range Ising models, Phys. Rev. B 92, 134204 (2015).

[67] R. Ketzmerick, K. Kruse, S. Kraut, and T. Geisel, What Determines the Spreading of a Wave Packet? Phys. Rev. Lett. 79, 1959 (1997).

[68] T. Ohtsuki and T. Kawarabayashi, Anomalous diffusion at the Anderson transitions, J. Phys. Soc. Jpn. 66, 314 (1997).

[69] Notice the correct normalization $\sum_{x} \Pi(x, t)=1$, and $\Pi(x, t) \geqslant 0$.

[70] V. Ros, M. Müller, and A. Scardicchio, Integrals of motion in the many-body localized phase, Nucl. Phys. B 891, 420 (2015).

[71] The single-particle mobility edges have been proven to exist at $W \gtrsim K[54]$.

[72] M. L. Mehta, Random Matrices, 3rd ed. (Elsevier Inc., Amsterdam, 2004).

[73] S. Bera, G. De Tomasi, I. M. Khaymovich, and A. Scardicchio, Return probability for the Anderson model on the random regular graph, Phys. Rev. B 98, 134205 (2018).

[74] G. de Tomasi, M. Amini, S. Bera, I. M. Khaymovich, and V. E. Kravtsov, Survival probability in generalized RosenzweigPorter random matrix ensemble, SciPost Phys. 6, 014 (2019).
[75] For larger fractions $f$ the dynamics at intermediate times will slow down due to the presence of the localized states across the mobility edge; please see Supplemental Note $S 1$ of [80] for different $f$.

[76] We define a fully ergodic phase as the phase in which the system can be described by the random matrix theory.

[77] In S2 note of [80] we explicitly show diffusive behavior in this disorder range $W<0.16 W_{\mathrm{AT}}$.

[78] A. Giacometti, Exact closed form of the return probability on the Bethe lattice, J. Phys. A 28, L13 (1995).

[79] For $W<0.16 W_{\mathrm{AT}}$ the propagation is diffusive with $\beta=1$ and consistent with the fully ergodic behavior given by Eq. (3).

[80] See Supplemental Material at http://link.aps.org/supplemental/ 10.1103/PhysRevB.101.100201 for additional data on different disorder strengths.

[81] $X_{\text {front }}(t)$ is given by the value of $x$ for which $\Pi(x, t)$ has a maximum.

[82] J. T. Edwards and D. J. Thouless, Numerical studies of localization in disordered systems, J. Phys. C: Solid State Phys. 5, 807 (1972).

[83] Note that both collapses, Eqs. (7) and (8), are consistent to each other provided the exponential behavior of $f(z)$ in Eq. (7), with the maximal value $\Pi\left(X_{\text {front }}(t), t\right)$ given by $g\left(X_{\text {front }}\right)[\Pi(0, t)-\Pi(0, \infty)]$ due to the normalization condition of $\Pi(x, t)$ (see Supplemental Note $S 4$ of [80] for more details).

[84] M. Serbyn, Z. Papić, and D. A. Abanin, Thouless energy and multifractality across the many-body localization transition, Phys. Rev. B 96, 104201 (2017).

[85] C. L. Bertrand and A. M. García-García, Anomalous Thouless energy and critical statistics on the metallic side of the many-body localization transition, Phys. Rev. B 94, 144201 (2016).

[86] In the literature [89-92] this kind of phase is usually called weakly ergodic when the eigenfunctions occupy a finite, but small fraction $f_{I} \ll 1$ of the Hilbert space, meaning that the system splits into a finite number $\sim 1 / f_{I}$ of nearly independent sectors which are ergodic within themselves, but not between each other.

[87] M. Aizenman and S. Warzel, Absolutely continuous spectrum implies ballistic transport for quantum particles in a random potential on tree graphs, J. Math. Phys. 53, 095205 (2012).

[88] D. J Luitz, I. M. Khaymovich, and Y. B. Lev, Multifractality and its role in anomalous transport in the disordered xxz spin-chain, arXiv: 1909.06380.

[89] E. Bogomolny and M. Sieber, Power-law random banded matrices and ultrametric matrices: Eigenvector distribution in the intermediate regime, Phys. Rev. E 98, 042116 (2018).

[90] P. A. Nosov, I. M. Khaymovich, and V. E. Kravtsov, Correlation-induced localization, Phys. Rev. B 99, 104203 (2019).

[91] P. A. Nosov and I. M. Khaymovich, Robustness of delocalization to the inclusion of soft constraints in long-range random models, Phys. Rev. B 99, 224208 (2019).

[92] A. Bäcker, M. Haque, and I. M. Khaymovich, Multifractal dimensions for random matrices, chaotic quantum maps, and many-body systems, Phys. Rev. E 100, 032117 (2019). 